\title{
Highs and lows
}

The rationale behind the frequent administration of chemotherapeutic drugs at low doses - high-time or metronomic dosing — is to prevent time for repair of damage to the tumour vasculature, thereby deriving increased therapeutic benefit. Logically, haematopoietic cells and gut epithelial tissues should also sustain more damage because of the lack of recovery time between cycles of chemotherapy, but, interestingly, such side effects, at least in the short term, seem to be much less severe. Bocci and colleagues provide an explanation for this as they show, in Cancer Research, that cycling endothelial cells are inherently more sensitive than other cells to continuous lowdose therapy, and suggest that this might therefore be an optimal way of delivering certain types of anti-angiogenic therapies, especially those using conventional chemotherapeutic drugs.

Bocci et al. exposed human tumour cells, fibroblasts and endothelial cells to daily low concentrations of chemotherapeutic drugs

\section{SENESCENCE}

\section{Stopping the cycle}

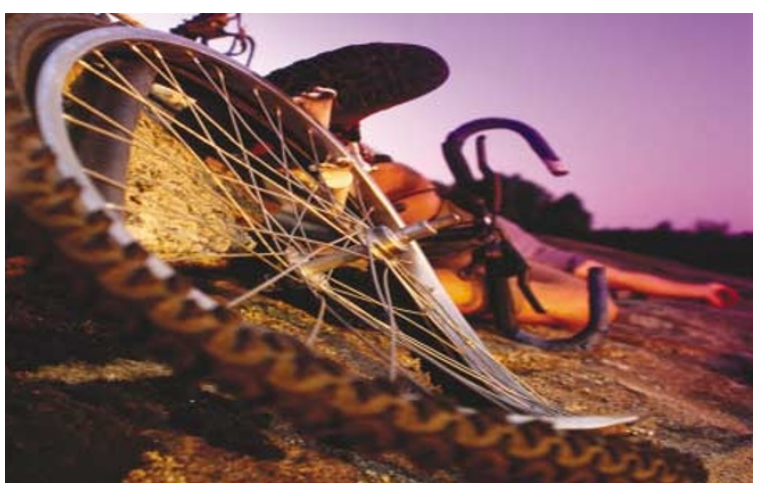

There are several different ways to stop cancercell proliferation. The most obvious approach is to induce apoptosis, and many chemotherapeutic agents are known to do just that. Less is known, however, about how to induce senescence - a state of terminal cell-cycle arrest in cancer cells.

In normal cells, replicative senescence results from loss of telomeric repeats after several rounds of cell division. This generates a DNAdamage signal that activates p53. Some anticancer drugs induce cell senescence by inducing for up to 6 days - analogous to the protracted metronomic protocols used in patients. They observed specific inhibition of endothelial-cell proliferation with taxanes and cyclophosphamide after 6 days, but not after 24 hours, using concentrations of 10-100 pM and 1-100 nM, respectively. By contrast, normal fibroblasts and breast cancer cell lines were not affected. Not all drugs showed this differential effect doxorubicin had similar antiproliferative activity against both endothelial cells and breast cancer cells. In addition, the authors observed that treated endothelial cells had a higher level of apoptosis than the cancer cell lines or fibroblasts.

So, prolonged exposure times, once an effective dose of drug has been reached, are crucial for cell kill in high-time chemotherapy regimens and might be selective for endothelial cells. This type of schedule might create an anti-angiogenic therapeutic window, and could be used to treat tumours that are resistant to the very
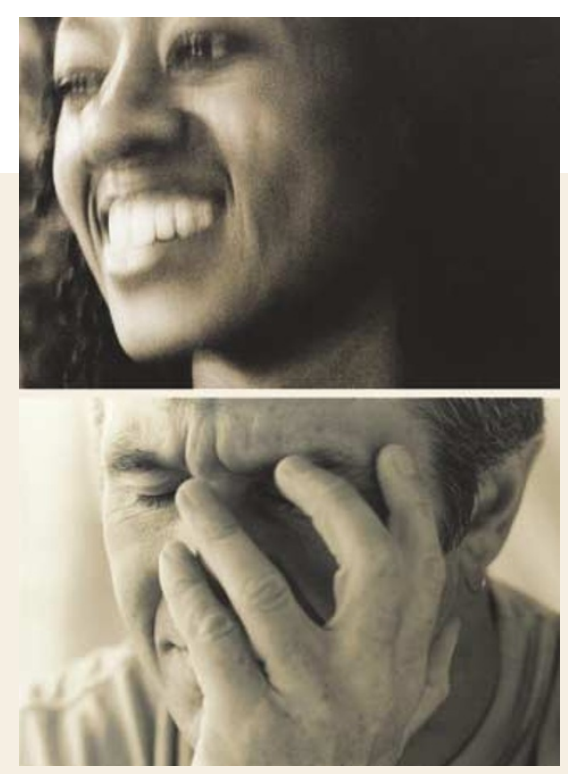

drugs that are used for low-dose chemotherapy or to decrease host toxicity without reducing efficacy. It remains to be seen whether other types of normal cells are also highly sensitive to metronomic dosing.

69) References and links Ezzie Hutchinson ORIGINAL RESEARCH PAPER Bocci, G. et al. Protracted low-dose effects on human endothelial cell proliferation and survival in vitro reveal a selective antiangiogenic window for various chemotherapeutic drugs. Cancer Res. 62. 6938-6943 (2002)

WEB SITE

Robert S. Kerbel's lab:

http://medbio.utoronto.ca/faculty/kerbel.htm this DNA-damage response in both normal and malignant cells, but these drugs can lead to unwanted side effects.

Dimitri Lodygin et al. therefore looked for signalling pathways that were specifically downregulated during replicative senescence. They reasoned that pharmacological inhibition of these pathways should induce senescence in tumour cells. Using microarray analysis, they found that several components of the cGMP signalling pathway were downregulated during replicative senescence of primary human diploid fibroblasts. So, could a compound such as 6-anilino-5,8-quinolinequinone (LY83583, or LY), which is known to inhibit cGMP production, induce senescence?

Lodygin et al. showed that treatment of fibroblasts with LY completely and irreversibly inhibited proliferation of the cells by blocking S-phase entry. Microarray analysis of LY-treated cells revealed a significant overlap between the genetic profiles of cells during replicative senescence and after LY treatment. One gene that was induced under both conditions was $C D K N 1 A$, which encodes an inhibitor of cyclin-dependent kinases - called WAF1 (also known as p21). LY also induced WAF1 expression and prevented proliferation in colorectal cancer, breast cancer and melanoma cell lines, indicating its therapeutic potential.
Experiments in cGMP-null cell lines revealed that LY's effects depended on induction of WAF1. LY did not, however, activate the DNA-damage/p53 pathway, as LY treatment of p53-deficient cells still resulted in WAF1 upregulation and senescence. It is not clear whether LY upregulation of WAF1 occurs through its effects on the cGMP signalling pathway, or through some other mechanism.

As LY induces cell-cycle arrest in a p53-independent manner, it might be useful in treating tumours that have p53 mutations. Inactivation of WAF1, on the other hand, has not been reported in cancer cells. In cells with disruptions in the RB pathway, LY induced apoptosis, rather than cellcycle arrest, so LY could also be used to treat tumours that have RB defects. The ability of LY to induce senescence without inducing DNA damage indicates that it might also have fewer side effects than current chemotherapeutic agents.

Kristine Novak

\section{(2) References and links}

ORIGiNAL ReSEARCH PAPER Lodygin, D., Menssen, A. \& Hermeking $\mathrm{H}$. Induction of the Cdk inhibitor 21 by LY 83583 inibits tumor cell pre 53-independent manner. J. Clin. Invest. 110, 1717-1727 (2002)

FURTHER READING Campisi, J. Cellular senescence as a tumor-suppressor mechanism. Trends Cell Biol. 11, 27-31 (2001)

\section{WEB SITE}

Heiko Hermeking's lab:

http://www.biochem.mpg.de/hermeking/ 